\title{
Structural Factor Governing Dyeability of Poly(ethylene terephthalate) Fibers
}

\author{
Kenji KAMIDE and Tomio KURIKI \\ Fundamental Research Laboratory of Fibers and Fiber-Forming \\ Polymers, Asahi Chemical Industry Co., Ltd., \\ 11-7 Hacchonawate, Takatsuki, Osaka 569, Japan
}

(Received May 16, 1986)

\begin{abstract}
An attempt was made to clarify whether there is a structural factor governing the dyeability of poly(ethylene terephthalate) (PET) fibers by disperse dye or not, and if so what it is. Five PET fiber samples having a wide degree of amorphous packing $(n)$ distribution, $F^{\prime}(n)$, as determined from dynamic loss tangent-temperature curves in an $\alpha_{\mathrm{a}}$ dispersion region using Manabe and Kamide's method, were prepared by changing spinning velocities and annealing conditions. PET fibers were dyed with the three different disperse dyes under various conditions and the dye uptake $W$ was determined colorimetrically. The upper limit of $n$, denoted as $n_{\mathrm{u}}^{\circ}$, above which the dye molecule can not penetrate through amorphous regions, was evaluated as functions of dyeing conditions from the plots of $W$ against $G\left(n_{\mathrm{u}}\right)\left(\equiv \int_{-\infty}^{n_{\mathrm{u}}} F^{\prime}(n) \mathrm{d} n\right)$ obtained for various assumed $n_{\mathrm{u}}$. It was confirmed that the plots of $W v s$. $G\left(n_{\mathrm{u}}^{\circ}\right)$ give a single master line, irrespective of the various fiber preparing conditions or dyeing conditions; for Resolin Blue FBL $W=67.6 \cdot G\left(n_{\mathrm{u}}^{\circ}\right)-0.4$ (mg-dye/gfiber). This indicates that the dyeability of PET fibers by the disperse dye is unambiguously governed by the amount of the amorphous region, whose degree of amorphous packing of $n$ is not larger than $n_{\mathrm{u}}^{\circ}$.

KEY WORDS Dyeability / Disperse Dye / Structural Factor / Dye Uptake / Degree of Amorphous Packing Distribution / Viscoelastic Property / Poly(ethylene terephthalate) /
\end{abstract}

The structural factors controlling the dyeability of PET fibers by the disperse dyes have not yet been systematically studied: Kitamura et al. ${ }^{1}$ found that (1) the penetration coefficient of a dye in heat-treated PET fibers increased with the intensity of the Xray small-angle scattering and the gauche content of the ethylene glycol unit in the amorphous region, and (2) with an increase in the temperature or time of heat-treatment, the X-ray intensity increased and the gauche content decreased. Yonetake et al. ${ }^{2}$ applied the mosaic block model for the dyeability of heattreated polypropylene films, and found that the crystallinity and the crystalline size especially in the direction of chain axis, as determined by the X-ray diffraction method, increased with heat-treatment and concluded that dye molecules were adsorbed more in the amorphous side region located between cores parallel to the chain axis than in the amorphous end region between lamella surfaces. However, note that these studies lack in the direct evaluation of the fine structure.

In the previous note, ${ }^{3}$ we demonstrated that the dyeability of poly(ethylene terephthalate) (PET) fibers by a disperse dye at 100-130 ${ }^{\circ} \mathrm{C}$ has a close relationship against the maximum value of the mechanical loss tangent, $(\tan \delta)_{\max }$, in their microbrownian movement $\left(\alpha_{\mathrm{a}}\right)$ regions and the temperature $T_{\max }$, at which $(\tan \delta)_{\max }$ is attained. Both parameters can be experimentally determined with good accuracy from the dynamic viscoelasticity $v s$. temperature $(T)$ curve in the $\alpha_{\mathrm{a}}$ dispersion region. Recently, Manabe and 
Kamide defined the second order structure characterized by the aggregation state of a polymer chain in a hypothetical cubic body with one side about $10 \mathrm{~nm}$ in length. ${ }^{4}$ They also introduced the degree of amorphous packing, $n$, of polymer segments for the second-order element, whose peak temperature of $\tan \delta$ is $T_{\max }^{\prime}$, by

$$
n=\left(T_{\max }^{\prime}-T_{\max }\right) /\left(\Delta T_{1 / 2}\right)_{\mathrm{R}}
$$

where $\left(\Delta T_{1 / 2}\right)_{\mathrm{R}}$ is the half-value width of the $\tan \delta-T$ curve of the second-order element. They showed theoretically that the distribution function $F^{\prime}(n)$ of the degree of amorphous packing, $n$, can be directly estimated from $\tan \delta-T$ curve in the $\alpha_{\mathrm{a}}$ dispersion. Completely amorphous polymers, including atactic polystyrene, have very narrow $n$ distributions, but semicrystalline polymers generally show significantly broad $n$ distributions. PET fibers obviously belong to the latter category. Alternatively, $n$ is also defined by the equation $1^{\prime}$

$$
n=\frac{\bar{f}-f}{\Delta \alpha\left(\Delta T_{1 / 2}\right)_{\mathrm{R}}}
$$

where $f$ is the free volume fraction, $\bar{f}, f$ at $n=0$, and $\Delta \alpha$ is the difference in the volume expansion coefficients above and below $T_{\mathrm{g}}$. Therefore, the dye molecule expected to penetrate preferentially into the region with lower degree of amorphous packing by the segmental motions of amorphous chains and in consequence, the dyeability should be governed by $F^{\prime}(n)$. This article confirms the above expectation by experimental results.

\section{THEORETICAL BACKGROUND}

According to Manabe and Kamide's theory, ${ }^{4,5}$ the region having the degree of amorphous packing of $n$ lower than a specific value $n_{\mathrm{u}}, G\left(n_{\mathrm{u}}\right)$, is given by

$$
G\left(n_{\mathrm{u}}\right)=\int_{-\infty}^{n_{\mathrm{u}}} F^{\prime}(n) \mathrm{d} n
$$

where $F^{\prime}(n)$ is normalized for a unit mass of the polymer sample.

If we suppose that the dye molecule can diffuse only into the region related to $G\left(n_{\mathrm{u}}^{\circ}\right)$ having $n$ not larger than $n_{\mathrm{u}}^{\circ}$, the dye uptake $W$ should be linearly proportional to $G\left(n_{\mathrm{u}}^{\circ}\right)$ :

$$
W=k \cdot G\left(n_{\mathrm{u}}^{\circ}\right)
$$

where $k$ is a proportional constant.

If the dye uptake for unit mass of a polymer sample possibly changes with $n$, then $k$ should be expressed as a function of $n, k(n)$, and eq 3 can be generalized in the form,

$$
W=\int_{-\infty}^{n_{\mathrm{u}}} k(n) \cdot F^{\prime}(n) \mathrm{d} n
$$

Analysis of the experimental data according to eq 4 is very difficult without detailed knowledge on $k(n)$.

Under the following conditions

$$
\begin{aligned}
& k(n)=k, n \leqq n_{\mathrm{u}}^{\circ} \\
& k(n)=0, n>n_{\mathrm{u}}^{\circ}
\end{aligned}
$$

eq 4 reduces readily to eq 3 and $n_{\mathrm{u}}^{\circ}$ can be determined in the manner that $W$ is proportional to $G\left(n_{\mathrm{u}}^{\circ}\right)$. $n_{\mathrm{u}}^{\circ}$ varies with dyeing temperature $T_{\mathrm{d}}$, dyeing time $t_{\mathrm{d}}$ and the molecular structure of the dye employed and is independent of $F^{\prime}(n)$. This procedure was successfully applied in part by Nakayama et al. ${ }^{6}$ in their study on the dyeing of polyacrylonitrile fibers. When eq 3 is acceptable, a master curve should be obtained between $W$ and $G\left(n_{\mathrm{u}}^{\circ}\right)$ for various fiber samples, dyed under different conditions.

\section{EXPERIMENTAL}

Five PET filaments (75 denier/36 filaments), melt spun from PET chips of the viscosity-average molecular weight $\bar{M}_{v}=$ $4.4 \times 10^{4}$ at various spinning velocities ranging $1.5-8.0 \times 10^{3} \mathrm{~m} \mathrm{~min}^{-1}$, were utilized. The preparing conditions of these samples are summarized in Table I. The detailed prepar- 
Table I. Conditions for the preparation of poly(ethylene terephthalate) filaments (viscosity-average molecular weight $\bar{M}_{v}=4.4 \times 10^{4}, 75$ denier/36 filaments) with different amorphous structures

\begin{tabular}{|c|c|c|c|c|c|}
\hline \multirow{2}{*}{$\begin{array}{l}\text { Sample } \\
\text { code }\end{array}$} & \multirow{2}{*}{$\begin{array}{l}\text { Spinning process } \\
\text { velocity } / \mathrm{m} \mathrm{min}^{-1}\end{array}$} & \multicolumn{2}{|c|}{ Annealing process } & \multicolumn{2}{|c|}{ Drawing process } \\
\hline & & Temperature, $T_{\mathrm{a}} /{ }^{\circ} \mathrm{C}$ & Time, $t_{\mathrm{a}} / \mathrm{s}$ & Draw ratio & Temperature $/{ }^{\circ} \mathrm{C}$ \\
\hline PD-1 & 4000 & 250 & 1 & - & - \\
\hline PD-2 & 8000 & 250 & 1 & - & - \\
\hline PD-3 & 7000 & 170 & 1 & - & - \\
\hline PD-4 & 5000 & 170 & 1 & - & - \\
\hline PD-5 & 1500 & - & - & 3.9 & 130 \\
\hline
\end{tabular}

ing procedure has already been described elsewhere. ${ }^{7}$ The as-spun filaments were heattreated at 170 or $250^{\circ} \mathrm{C}$ for $1 \mathrm{~s}$, using a special annealing apparatus, designed and constructed before, ${ }^{7}$ or drawn at $130^{\circ} \mathrm{C}$ to give five samples differing greatly in $F^{\prime}(n)$.

For the samples $\tan \delta-T$ curves were determined using a Rheovibron DDV-IIc at $110 \mathrm{~Hz}$ and at a heating rate of $10 \mathrm{~K} \mathrm{~min}^{-1}$. By examination of the curve obtained using Manabe and Kamide's method, ${ }^{4} F^{\prime}(n)$ was evaluated.

Three disperse dyes were utilized: Resolin Blue FBL (RBF) (1,8-dihydroxy-2-bromo4,11-diamino-anthraquinone, molecular weight $=349$, manufactured by Bayer AG (Leverkusen DBR)), Diacelliton Fast Orange GL (DFO) (4-nitro-4'-amino-azobenzene, molecular weight $=242$, manufactured by Mitsubishi Chemical Co., Ltd.), and Miketon Fast Red R (MFR) (4-nitro-4' $(N, N$-dihydroxyethyl)-amino-azobenzene, molecular weight $=$ 344, manufactured by Mitsui-Toatsu Chemical Co., Ltd.). These dyes were purified by recrystallization from their acetone solutions. The molecular structures of the dyes are shown in Figure 1. DFO and MFR are similar in chemical structure and differences in dyeability, if any, may be due to molecular weight difference. RBF and MFR have almost the same molecular weight, but differ in molecular shape. The PET samples were dyed under the following conditions: dye concentration, $1.2 \mathrm{gl}^{-1}$; liquor ratio, 200; dyeing
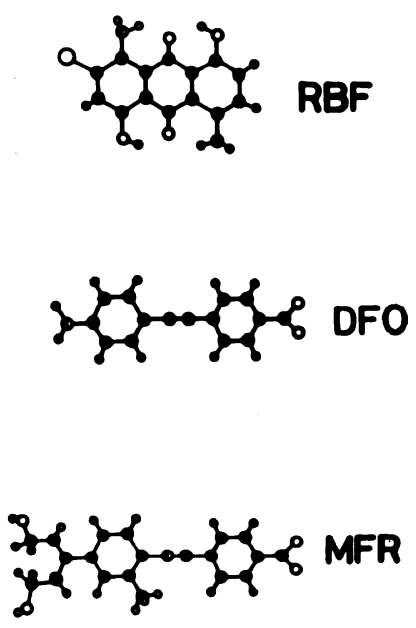

Figure 1. Chemical structures of three dyes utilized in this study: RBF, Resolin Blue FBL; DFO, Diacelliton Fast Orange GL; MFR, Miketon Fast Red R. Filled circle, carbon atom; small unfilled circle, hydrogen atom; middle unfilled circle, oxygen atom; half-filled circle, nitrogen atom; large unfilled circle, bromine atom.

temperature $\left(T_{\mathrm{d}}\right), 90,100,110$, and $130^{\circ} \mathrm{C}$; dyeing time $\left(t_{\mathrm{d}}\right), 30-360 \mathrm{~min}$.

The dyed sample $(0.1 \mathrm{~g})$ was twice washed with distilled water at room temperature and then extracted with a $100 \mathrm{ml}$ of $N, N$-dimethylformamide (DMF) at $60^{\circ} \mathrm{C}$. The extraction procedure was repeated three times and the extracts were gathered and diluted with fresh DMF to give a $500 \mathrm{ml}$ solution. The total amount of dye absorbed by a fiber was colorimetrically determined on a Shimadzu UV-360 type spectrophotometer. 


\section{RESULTS AND DISCUSSION}

Figure 2 shows the $F^{\prime}(n)$ curve of PET fibers prepared in this study. Here, the $T_{\max }$ $\left(=128^{\circ} \mathrm{C}\right)$ of the sample code PD-5 was decided at $n=0$. The five samples have widely different $F^{\prime}(n)$ : for example, sample code PD-2 and PD-3 contain larger amounts of low $n$ region than sample code PD-4. It has already been confirmed that $F^{\prime}(n)$ of the samples does not change during the dyeing process.

Figure 3 shows that dye uptake $W$ depends on $t_{\mathrm{d}}$. In the range of $t_{\mathrm{d}}<360 \mathrm{~min}, W$ does not reach its asymptotic value; at $T_{\mathrm{d}} \geqq 130^{\circ} \mathrm{C}$, the order of the magnitude of $W$ in five samples changes sometimes with $t_{\mathrm{d}}$.

Figure 4 shows some examples of the

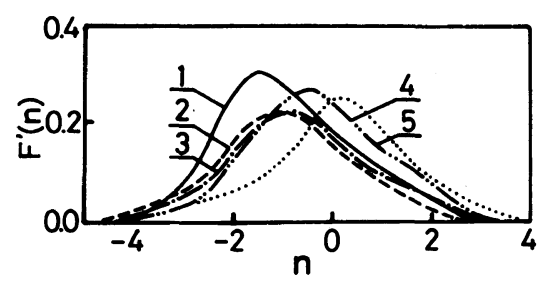

Figure 2. Normalized degree of amorphous packing distribution $F^{\prime}(n)$ of the amorphous region of PET fibers: 1, sample code PD-1; 2, sample code PD-2; 3, sample code PD-3; 4, sample code PD-4; 5, sample code PD-5; see also Table I.

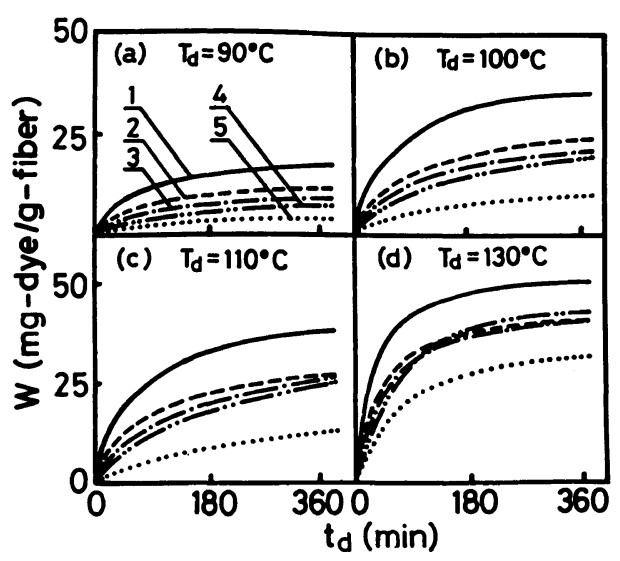

Figure 3. Change in the dye uptake $W$ of RBF with the dyeing temperature $T_{\mathrm{d}}$ and the dyeing time $t_{\mathrm{d}}$. Numbers on curves have the same meaning as those in Figure 2. relations between $W$ and $G\left(n_{\mathrm{u}}\right)$. The number in the figure is the assumed value of $n_{\mathrm{u}}$. For a specific $n_{\mathrm{u}}\left(n_{\mathrm{u}}^{\circ}\right), W$ is proportional to $G\left(n_{\mathrm{u}}\right)$. When the linear relationship between $W$ and $G\left(n_{\mathrm{u}}^{\circ}\right)$ holds, the $W$ value of intercept at $G\left(n_{\mathrm{u}}^{\circ}\right)=0$ should be zero. This intercept value of $W$ is given by the product of the slope, $\tan \alpha$, of the plot in Figure 4 and absolute value of an intercept at $W=0$, $\left|\lim _{W \rightarrow 0} G\left(n_{\mathrm{u}}^{\circ}\right)\right|$. Then the product of $\tan \alpha$. $\left|\lim _{W \rightarrow 0} G\left(n_{\mathrm{u}}^{\circ}\right)\right|$ can be employed as a measure of the deviation of the $W$ vs. $G\left(n_{\mathrm{u}}^{\circ}\right)$ plots from eq 3 .

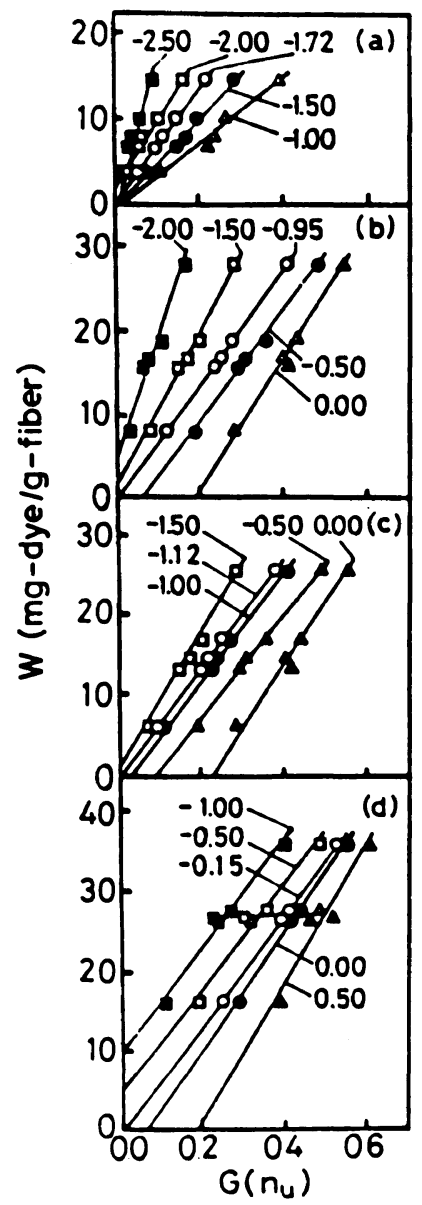

Figure 4. Plot of the dye uptake $W$ of RBF $v s . G\left(n_{\mathrm{u}}\right)$. a) $\left.T_{\mathrm{d}}=90^{\circ} \mathrm{C}, t_{\mathrm{d}}=360 \mathrm{~min} ; \mathrm{b}\right) T_{\mathrm{d}}=100^{\circ} \mathrm{C}, t_{\mathrm{d}}=360 \mathrm{~min}$; c) $T_{\mathrm{d}}=110^{\circ} \mathrm{C}, t_{\mathrm{d}}=240 \mathrm{~min} ;$ d) $T_{\mathrm{d}}=130^{\circ} \mathrm{C}, t_{\mathrm{d}}=120 \mathrm{~min}$. Numbers on the curves mean the assumed $n_{\mathrm{u}}$ values. 


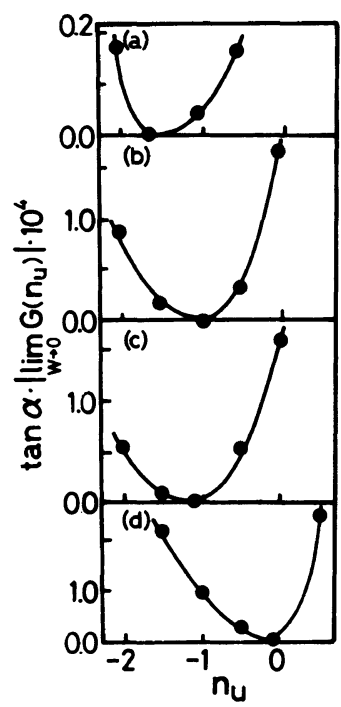

Figure 5. The production of $\tan \alpha \cdot\left|\lim _{W \rightarrow 0} G\left(n_{\mathrm{u}}\right)\right| \times 10^{4}$ plotted as a function of the assumed $n_{\mathrm{u}}$ for RBF. a) $T_{\mathrm{d}}=$ $90^{\circ} \mathrm{C}, t_{\mathrm{d}}=360 \mathrm{~min}$; b) $T_{\mathrm{d}}=100^{\circ} \mathrm{C}, t_{\mathrm{d}}=360 \mathrm{~min}$; c) $T_{\mathrm{d}}=$ $110^{\circ} \mathrm{C}, t_{\mathrm{d}}=240 \mathrm{~min}$; d) $T_{\mathrm{d}}=130^{\circ} \mathrm{C}, t_{\mathrm{d}}=120 \mathrm{~min}$.

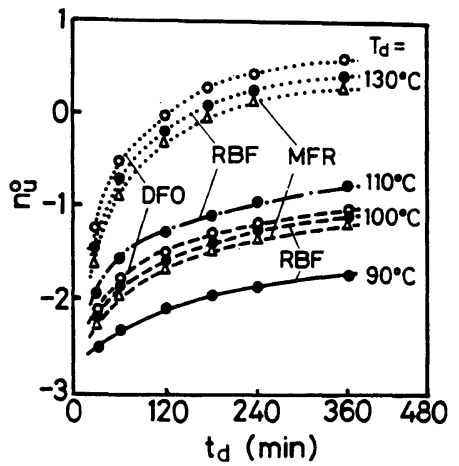

Figure 6. Change in $n_{\mathrm{u}}^{\circ}$ with dyeing time $t_{\mathrm{d}}$ : full line, $T_{\mathrm{d}}=90^{\circ} \mathrm{C}$; broken line, $T_{\mathrm{d}}=100^{\circ} \mathrm{C}$; chain line, $T_{\mathrm{d}}=$ $110^{\circ} \mathrm{C}$; dotted line, $T_{\mathrm{d}}=130^{\circ} \mathrm{C} ; \bigcirc, \mathrm{DFO} ; \mathrm{O}, \mathrm{RBF} ; \triangle$, MFR.

Figure 5 shows the plot of $\tan \alpha \cdot \mid \lim _{W \rightarrow 0}$ $G\left(n_{\mathrm{u}}^{\circ}\right) \mid v s . n_{\mathrm{u}}$ constructed from the data in Figure 4. The $n_{\mathrm{u}}$ value corresponding to the minimum of the product is the true $n_{\mathrm{u}}^{\circ}$. The $n_{\mathrm{u}}^{\circ}$ values for other dyeing conditions, not shown in Figures 3 and 4, was also determined using the present method.

Figure 6 shows the effects of the dyeing conditions $\left(T_{\mathrm{d}}\right.$ and $\left.t_{\mathrm{d}}\right)$ on $n_{\mathrm{u}}^{\circ}$. The dye mol-

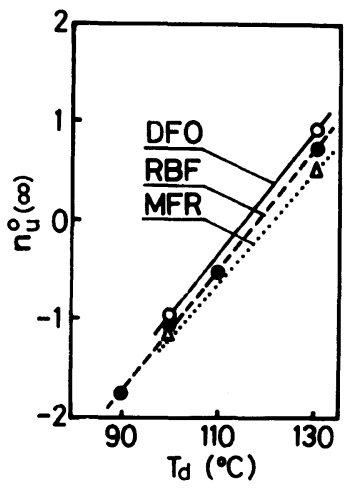

Figure 7. Relations between $n_{\mathrm{u}}^{\circ}(x)$ and dyeing temperature $T_{\mathrm{d}}$.

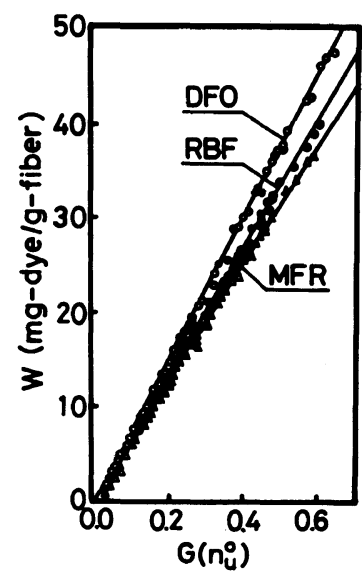

Figure 8. Experimental relations between the dye uptake $W$ and $G\left(n_{\mathrm{u}}^{\circ}\right)$ for PET fibers.

ecule with larger molecular weight penetrates into the larger $n$ region as the sample is dyed at higher temperature for a longer period. The $t_{\mathrm{d}}$ dependence of $n_{\mathrm{u}}$ in shorter $t_{\mathrm{d}}$ becomes remarkable at higher $T_{\mathrm{d}}$.

We estimated $n_{\mathrm{u}}^{\circ}$ at infinite time of $t_{\mathrm{d}}$, $n_{\mathrm{u}}^{\circ}(\infty)$, by extrapolating the curves in Figure 6 to the infinite value of $t_{\mathrm{d}}$ and plotted it as a function of $T_{\mathrm{d}}$ in Figure 7 . The $n_{\mathrm{u}}^{\circ}(\infty)$ value increases linearly with $T_{\mathrm{d}}$. At higher $T_{\mathrm{d}}$, the dye can penetrate into a more closely packed region of the amorphous phase. $n_{\mathrm{u}}^{\circ}(\infty)$ decreases in the following order at a given $T_{\mathrm{d}}$ : $\mathrm{DFO}>\mathrm{RBF}>\mathrm{MFR}$.

Figure 8 summarizes the relations between 

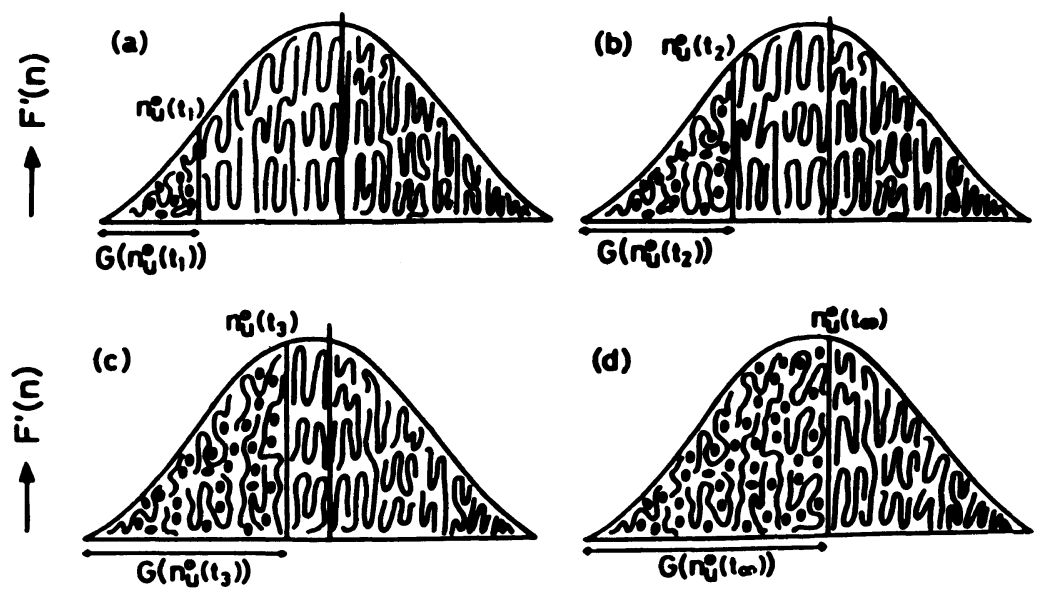

Figure 9. Schematic representation of the dyeing phenomena of amorphous region, represented by $F^{\prime}(n)$, as a function of dyeing time $t_{\mathrm{d}}$. Black circles indicate dye molecules.

$W$ and $G\left(n_{\mathrm{u}}^{\circ}\right)$, obtained for various samples dyed under various conditions. All the experimental points for each dye fall on a single straight master line expressed by

$$
\begin{array}{lll}
W=67.6 \cdot G\left(n_{\mathrm{u}}^{\circ}\right)-0.4 & \text { for } & \mathrm{RBF} \\
W=75.3 \cdot G\left(n_{\mathrm{u}}^{\circ}\right)-0.4 & \text { for } & \mathrm{DFO} \\
W=63.0 \cdot G\left(n_{\mathrm{u}}^{\circ}\right)-0.3 & \text { for } & \mathrm{MFR}
\end{array}
$$

using the least-squares method. The second term of the right-hand side in eq 6 can be neglected with good approximation as compared with the first term of the same side; then eq 6 can be regarded as an experimental expression for eq 3. Figure 6 indicates that $k$ in eq 3 is constant for a given dye, irrespective of various PET samples and the dyeing conditions. Different $k$ values were obtained for different dye molecules.

As long as disperse dyes are utilized, the dyeability of PET fibers is unambiguously determined by the amount of amorphous region, where the degree of amorphous packing $n$ is not larger than $n_{\mathrm{u}}^{\circ}$.

The slope of the plots in Figure 8 is not a single function of the molecular weight of the dye, but depends on the general shape of the dye molecule and probably on the PET molecule-dye molecule interaction. For example,
RBF and MFR have similar molecular weight $(345 \pm 5)$. Note that the axial ratio of the RBF molecule is not far from unity, but that of MFR is larger than 3. It is clear that the PET (and probably any) fibers can be dyed easily with dyes of smaller molecular weights and compact form.

On the basis of the experimental results obtained here, we can speculate the detailed mechanism of dyeing of PET fibers with disperse dye. Figure 9 shows a schematic representation of the dyeing phenomena of the amorphous region as a function of dyeing time $t_{\mathrm{d}}$. In the figure, the black circle is the dye molecule. Explanations for Figure 9 are given as follows.

(1) At given $T_{\mathrm{d}}$, the PET molecules in the amorphous region not larger than $n_{\mathbf{u}}^{\circ}$ have vigorous microbrownian movement and this value of $n_{\mathrm{u}}^{\circ}$ coincides with $n_{\mathrm{u}}^{\circ}(\infty)$ as denoted by full vertical line in the figure. When some thermodynamic interactions between PET chains and water occur (in fact, the significant effects of water on the glass transition ${ }^{8}$ and the melting ${ }^{9}$ of PET were confirmed by DSC studies), $F^{\prime}(n)$ for the wet PET fiber in the dyeing process may deviate from $F^{\prime}(n)$ for the dry PET solid. Thus, $n_{\mathrm{u}}^{\circ}(\infty)$ in Figure 9 does not always coincide with $n_{\mathrm{u}}^{\circ}$ for infinite $t_{\mathrm{d}}$ at 
given $T_{\mathrm{d}}$ estimated for the dry PET solid. Unfortunately, attempts to estimate $F^{\prime}(n)$ for PET in water were not successful.

(2) Only the amorphous region belonging to the fraction between $n_{\mathrm{u}}^{\circ}\left(t_{2}\right)$ and $n_{\mathrm{u}}^{\circ}\left(t_{1}\right)$ is dyed during the dyeing time between $t_{2}$ and $t_{1}$.

(3) The dye uptake $W$ by the amorphous region with $n_{\mathrm{u}}<n_{\mathrm{u}}^{\circ}\left(t_{1}\right)$ (i.e., the region already dyed during $t_{1}$ ) is maintained constant during further dyeing $\left(t_{\mathrm{d}}>t_{1}\right)$.

(4) PET molecules in the amorphous region with higher $n_{\mathrm{u}}$ and those in the crystalline region are not dyed. The latter can be experimentally confirmed by the fact that the crystallinity, crystal size and crystal orientation of PET fibers evaluated by X-ray diffraction method do not change before and after dyeing.

In order to avoid confusion and misunderstanding, it should be noted that Figure 9(a) - (c) does not mean a one-dimensional diffusion of the dye from lower $n_{\mathrm{u}}$ to higher $n_{\mathrm{u}}$. As described before, we defined the secondorder structural elements as the aggregation of uniform polymeric chains, on the basis of segmental microbrownian movement. The second-order structural elements for a given polymer solid sample are separated from each other and arranged in the order of increasing $n_{\mathrm{u}}$. The results indicate the $F^{\prime}(n)$ value shown in Figure 2. Obviously, an increase in $n_{\mathrm{u}}^{\circ}$ with $t_{\mathrm{d}}$ as a result of diffusion is expected to cause a rather three-dimensional nature of the dye molecules.

The amorphous regions having different $n$ bring about a dramatic increase in the free volume fraction by occurrence of microBrownian movement above their $T_{\mathrm{g}}$. The fractional free volume is generally given by

$$
f=f_{\mathrm{g}}+\Delta \alpha\left(T-T_{\mathrm{g}}\right)
$$

where $f_{\mathrm{g}}$ is $f(\cong 0.025)$ at $T_{\mathrm{g}}$.

From eq $1^{\prime}$ and $7, n$ is obviously governed by the temperature $T$ in a $\tan \delta-T$ curve in $\alpha_{\mathrm{a}}$ dispersion region and $f$ is also closely related with $T_{\mathrm{g}}$, as pointed out previously. Equation
7 means that the free volume fraction at temperature above $T_{\mathrm{g}}$ is larger for larger $n$.

Commercially available PET fibers have a degree of amorphous packing similar to sample code PD-5. The dye uptake of this sample, dyed at $130^{\circ} \mathrm{C}$ for $60 \mathrm{~min}$, was 14.5 mg-dye/g-fiber for RBF, $16.5 \mathrm{mg}$-dye/g-fiber for DFO and $12.8 \mathrm{mg}$-dye/g-fiber for MFR, which correspond to $G\left(n_{\mathrm{u}}^{\circ}\right)$ value of 0.21 , 0.23 , and 0.19 , respectively. This means that when PET fibers having $G\left(n_{\mathrm{u}}^{\circ}\right)$ larger than 0.21 at given $T_{\mathrm{d}}$ are dyed with RBF for $60 \mathrm{~min}, n_{\mathrm{u}}^{\circ}$ is a function of $T_{\mathrm{d}}$ and $t_{\mathrm{d}}$ (see Figure 6), and $n_{\mathrm{u}}^{\circ}$ at $t_{\mathrm{d}}=60$ min can be determined experimentally with a given dye for a given $T_{\mathrm{d}}$. The preparing conditions for the PET fiber samples with $G\left(n_{\mathrm{u}}^{\circ}\left(100^{\circ} \mathrm{C}\right)\right)>0.21$ were examined using variously prepared samples in our previous papers. $^{3,10,11}$ We found, for example, that the PET fibers spun with a spinning velocity ranging from 4.5 to $8.0 \mathrm{~km} \mathrm{~min}^{-1}$ and then annealed at a temperature $T_{\mathrm{a}}$ ranging from 250 to $255^{\circ} \mathrm{C}$ at a $-5-+5 \%$ extension have never been dyed with RBF at $90^{\circ} \mathrm{C}$. Then, in order to confirm the reliability of our theoretical prediction, these samples were dyed at $95^{\circ} \mathrm{C}$ with $\mathrm{RBF}$ and $W$ was evaluated to be $12.3-$ $13.6 \mathrm{mg}$-dye/g-fiber.

These results indicate that PET fibers are dyeable by commercially available disperse dyes even at $95^{\circ} \mathrm{C}$, if the amorphous supermolecular structure of the fibers controlled in advance.

In conclusion, the parameter $G\left(n_{\mathrm{u}}^{\circ}\right)$, defined in this article, can be regarded as an important structural factor governing the dyeability of PET fibers.

\section{REFERENCES}

1. K. Kitamura, F. Shibata, and Z. Yoshida, Sen-i Gakkaishi, 8, 359 (1971).

2. K. Yonetake, T. Masuko, T. Shimanuki, and M. Karasawa, J. Appl. Polym. Sci., 28, 3049 (1983).

3. K. Kamide, T. Kuriki, and S. Manabe, Polym. J., 18, 167 (1986).

4. S. Manabe and K. Kamide, Polym. J., 16, 375 (1984).

5. S. Manabe, K. Kamide, C. Nakayama, and S. 


\section{K. KAMIDE and T. KURIKI}

Kobayashi, J. Text. Mach. Soc. Jpn., 30, T85 (1977), and also J. Text. Mech. Soc. Jpn., English Edition, 27, 10 (1981).

6. C. Nakayama, K. Kamide, and S. Manabe, Sen-i Gakkaishi, 33, T-192 (1977).

7. K. Kamide, T. Kuriki, and S. Manabe, Polym. J., 18, 163 (1986).

8. T. Hatakeyama and H. Hatakeyama, Sen- $i$
Gakkaishi, 39, T461 (1983).

9. K. Kamide, T. Kuriki, and M. Saito, unpublished results.

10. T. Kuriki, S. Manabe, and K. Kamide, J. Text. Mach. Soc. Jpn., 38, T150 (1985).

11. T. Kuriki, K. Kamide, and Y. Komatsu, J. Text. Mach. Soc. Jpn., 38, T192 (1985). 